\title{
ANÁLISE ESPACIAL DE ATRIBUTOS QUÍMICOS DO SOLO E DA PRODUÇÃO DA CULTURA PIMENTA-DO-REINO (PIPER NIGRUM, L.)
}

\author{
SPATIAL ANALYSIS OF CHEMICAL ATTRIBUTES OF THE SOIL AND \\ PRODUCTIVITY IN THE BLACK PEPPER CROP (PIPER NIGRUM, L.)
}

Julião Soares de Souza Lima ${ }^{*}$; Rone Batista de Oliveira ${ }^{1}$; Wadson da Rocha²;

Paulo César Oliveira ${ }^{1}$; Waylson Zancanella Quartezani ${ }^{1}$

\section{RESUMO}

$\mathrm{O}$ conhecimento de determinados atributos do solo associado à resposta de produção de pimenta-do-reino pode possibilitar a aplicação racional, localizada e individualizada dos insumos. O objetivo deste trabalho foi avaliar a variabilidade espacial dos atributos químicos e da produção de pimenta-do-reino. Os atributos químicos do solo foram amostrados na projeção da copa da cultura e na profundidade de 0-0,20 m, com distância entre as linhas de 2,50 m e de 4,0 m entre amostras na linha de plantio, totalizando 76 pontos amostrais. Em cada ponto, foram determinados os atributos $\mathrm{P}, \mathrm{K}, \mathrm{Ca}, \mathrm{Mg}, \mathrm{H}+\mathrm{Al}$ e acidez ativa (pH). Com base nessas análises, determinou-se a soma de cátions básicos (SB), capacidade de troca catiônica (CTC) e saturação por cátions básicos (V). Os atributos pH, P, K, Al, H+Al e produção apresentaram estrutura de dependência espacial, o que permitiu o seu mapeamento com a utilização de técnicas geoestatísticas. Os atributos químicos apresentaram valores baixos de alcance, com exceção da variável fósforo, evidenciando a baixa continuidade espacial do solo sob pimenta-do-reino. A grande amplitude de variação dos atributos químicos justifica a aplicação diferenciada e localizada de fertilizantes na lavoura.

Palavras chave: Manejo do solo, mapas, semivariograma.

\section{ABSTRACT}

The knowledge of determined attributes of the soil associated to the reply of productivity of the black pepper can make the application rational, located and individualized of the farm input. This work was carried out to evaluate the spatial variability of the chemical attributes and productivity in the black pepper. The soil chemical attributes were collected from under the projection of the plant canopy's to the depth of 0-0.20 m, with distance among lines of $2.5 \mathrm{~m}$ and $4.0 \mathrm{~m}$ among feet in the planting line, totaling 76 points. In each point were determinate the attributes $P, K, C a, M g, H+A l$ and activates acidity $(\mathrm{pH})$. With base in those analyses, the sum of basic cations $(S B)$, capacity of cationic change (CTC) and basic saturation $(V)$ were calculated. The attributes $p H, P, K, A l$, $H+A l$ and production presented a spatial dependence structure, allowing their mapping by geostatistics techniques. Chemical attributes presented low range values, exception the $P$ attribute, evidencing the low soil continuity spatial under the black pepper crop. The wide variation range of chemical attributes shows the necessity of individualized points of fertilizer application for black pepper cropping.

Key words: Soil management, maps, semivariograms.

1 Departamento de Engenharia Rural, Universidade Federal do Espírito Santo - UFES. Alegre-ES, Brasil. Cx postal: 16, CEP: 29500-00, E-mail: limajss@yahoo.com.br.

2 Pesquisador do CNPGL - EMBRAPA. Juiz de Fora-MG.

* Autor para correspondência. 


\section{INTRODUÇÃO}

A pimenta-do-reino, também conhecida como pimenta-da-Índia, é uma planta trepadeira de grande produtividade e uma das mais valorizadas condimentares do mundo, com grande valor econômico, permitindo que a atividade desenvolvida pelos pipericultores seja altamente rentável.

O Brasil é um dos maiores produtores de pimenta-do-reino, oscilando entre a segunda e terceira posição no mercado mundial. Das 50 mil toneladas por ano, o país exporta $45 \mathrm{mil}$, principalmente para a Europa e para os Estados Unidos. O Estado do Espírito Santo é o segundo maior produtor de pimenta-do-reino do Brasil, responsável por $18 \%$ da produção nacional. O cultivo capixaba do produto é concentrado essencialmente no município de São Mateus, região norte, que responde por $73,5 \%$ da produção (Secundino, 2003).

O conhecimento da nutrição da pimenta-doreino em décadas anteriores era bastante incipiente, não tendo sido, ainda, determinados os distúrbios ocasionados pelas deficiências ou excessos de nutrientes (Veloso, 1993).

Atualmente, a cultura da pimenta-do-reino recebe um manejo convencional, no qual as práticas culturais são aplicadas uniformemente, com base na média, considerando a homogeneidade dos fatores de produção em todo o campo. Porém, o desconhecimento pontual desses fatores pode proporcionar metas impróprias de produtividade com uso excessivo de insumos, tornando a produção ineficiente com altos custos e danos ambientais.

O conhecimento da variação de atributos químicos do solo é importante para o seu manejo, planejamento de esquemas de amostragem e gerenciamento de práticas agrícolas. Antes de buscar qualquer relação destes elementos com a cultura, é importante avaliar a extensão e a intensidade da dependência espacial desta variação, isoladamente ou em conjunto com outros parâmetros (Gandah et al., 2000).

Considerando que os fenômenos naturais não podem ser tratados como fenômenos aleatórios, faz-se necessário utilizar ferramentas matemáticas que permitem estudar as duas características essenciais das variáveis aleatórias, ou seja, o aspecto aleatório e o espacial. Para este estudo utilizam-se as técnicas da geoestatística, que são muito empregados na agricultura de precisão, que permite estudar a dependência espacial de atributos do solo e os relativos às plantas considerando a distância entre as amostras, permitindo a interpretação e projeção dos resultados com base na estrutura da sua variabilidade natural (Vieira, 2000 e Ortiz, 2003).

O uso de técnicas de agricultura de precisão com a finalidade de se mapear e manejar a variabilidade de atributos de fertilidade do solo introduz um fato novo nas lavouras, na medida em que se deixa de considerar determinadas áreas agrícolas como uniformes para dividi-las em pequenos talhões ou zonas de manejo, que, por possuírem características próprias e serem determinantes sobre os índices de produtividades obtidas, passam a serem analisados individualmente quanto ao tipo e qualidade de nutriente a receber (Saraiva et al., 2000).

A aplicação da agricultura de precisão na cultura da pimenta-do-reino ainda não é uma realidade na agricultura brasileira, uma vez que existem poucos ou nenhum trabalho com esta abordagem. Portanto, o objetivo deste trabalho foi caracterizar a variabilidade espacial de atributos químicos do solo e da produção da cultura da pimenta-do-reino na região norte do Estado do Espírito Santo - Brasil.

\section{MATERIAL E MÉTODOS}

O experimento foi realizado numa área comercial de produção de pimenta-do-reino no município de São Mateus, região norte do Estado do Espírito Santo - Brasil, situada na latitude $18^{\circ} 42^{\prime} \mathrm{S}$ e longitude $39^{\circ}$ $51^{\prime} \mathrm{W}$, numa altitude de $26 \mathrm{~m}$ sobre o nível do mar, sob temperatura média anual de $24^{\circ} \mathrm{C}$ com máximas diárias de $29,3^{\circ} \mathrm{C}$ e mínimas de $20,8^{\circ} \mathrm{C}$.

$\mathrm{O}$ solo da área foi classificado como Latossolo Vermelho-Amarelo distrófico (Embrapa, 1999), com as frações texturais do solo em 63,9\% de areia grossa; $11,4 \%$ de areia fina; $8,2 \%$ de silte e $16,3 \%$ de argila. A variedade de pimenteira cultivada é a Bragantina com quatro anos de idade, plantada no espaçamento 2,5 x 2,0 m (2000 plantas ha-1) em fileiras simples, e sob regime de irrigação por aspersão. O sistema de manejo adotado pelo agricultor constitui-se de quatro colheitas por ano, em média, com controle de plantas invasoras, aplicação de herbicidas e reposição de nutrientes no solo realizado dois meses após cada colheita.

Para o estudo selecionou-se no centro da lavoura uma área de 0,1 ha, com 12,5 metros de largura (6 linhas: A, B, C, D, E e F) por 80 metros de comprimento (40 colunas). Considerando os 
efeitos de bordadura eliminaram-se as linhas A e F, bem como a primeira e última coluna, ficando a área útil para as correlações delimitada por um grid de amostragem de $780 \mathrm{~m}^{2}$, com 152 pontos amostrais (plantas).

A colheita da cultura foi realizada no mês de agosto de 2004. Os cachos colhidos, manualmente, foram acondicionados em sacos de papel e posteriormente determinada a massa de cada amostra com a utilização de uma balança analítica de precisão. Para a determinação da produção por planta considerou-se a massa verde dos grãos.

Os atributos químicos do solo foram amostrados na projeção da copa da cultura e na profundidade de $0-0,20 \mathrm{~m}$, com distância de quatro metros entre amostras na linha de plantio totalizando 76 pontos. Nessas amostras foram feitas análises para determinação dos teores de fósforo $(\mathrm{P})$, potássio $(\mathrm{K})$, cálcio $(\mathrm{Ca})$, magnésio $(\mathrm{Mg})$, acidez potencial $(\mathrm{H}+\mathrm{Al})$ e acidez ativa ( $\mathrm{pH}$ em água), conforme preconizada pela Embrapa (1997). Com base nessas análises, calcularam-se a soma de cátions básicos (SB), capacidade de troca catiônica (CTC) e saturação por cátions básicos $(\mathrm{V} \%)$.

Os dados foram inicialmente avaliados por meio da estatística descritiva (média; mediana; variância; desvio padrão, coeficiente de variação, simetria e curtose). Para a verificação dos dados candidatos a "outliers" foram analisados os quartis superiores e inferiores das freqüências (Hoaglin et al., 1983). A hipótese de normalidade dos dados foi testada pelo teste Kolmogorov-Smirnov-KS. Em seguida realizou-se a análise exploratória, dentro da qual se buscou avaliar o atendimento da hipótese de estacionariedade assumida, como descrito por Gonçalves et al. (2001) e Lima et al. (2007).

A análise da dependência espacial foi avaliada com bases nas pressuposições de estacionaridade da hipótese intrínseca, pela análise de semivariogramas ajustados pelo software $\mathrm{GS}^{+}$(Robertson, 1998). Para tal, foi utilizado o semivariograma clássico de Matheron, que é dado pela Equação 1.

$$
\gamma^{*}(\mathrm{~h})=\frac{1}{2 \mathrm{~N}(\mathrm{~h})} \sum_{i=1}^{\mathrm{N}(\mathrm{h})}\left[\mathrm{Z}\left(\mathrm{x}_{\mathrm{i}}\right)-\mathrm{Z}\left(\mathrm{x}_{\mathrm{i}}+\mathrm{h}\right)\right]^{2} 01
$$

em que $\mathrm{N}(\mathrm{h})$ é o número de pares experimentais de observações $\mathrm{Z}\left(\mathrm{x}_{\mathrm{i}}\right), \mathrm{Z}\left(\mathrm{x}_{\mathrm{i}}+\mathrm{h}\right)$ separados por um vetor h. O semivariograma é representado pelo gráfico $\gamma^{*}$ (h) versus h. No ajuste dos modelos teóricos (esféricos, exponencial, linear e gaussiano) aos semivariogramas experimentais determinou-se os coeficientes efeito pepita $\left(\mathrm{C}_{0}\right)$, patamar $\left(\mathrm{C}_{0}\right.$ $\left.+\mathrm{C}_{1}\right)$, variância estrutural $\left(\mathrm{C}_{1}\right)$ e alcance $(\mathrm{a})$. Os modelos foram escolhidos segundo a menor soma do quadrado dos resíduos (SQR), maior coeficiente de determinação $\left(R^{2}\right)$ e o maior coeficiente de correlação entre o valor conhecido e o estimado pelo modelo na validação cruzada.

Para análise do índice de dependência espacial (IDE) dos atributos, foi utilizada a relação $\mathrm{C}_{1} /\left(\mathrm{C}_{0}+\mathrm{C}_{1}\right)$, definido no software e classificado por Zimback (2001) como forte dependência para IDE maior que $75 \%$, moderada dependência entre $25 \%$ a $75 \%$ e baixa dependência para relação menor que $25 \%$. Comprovada a dependência a espacial realizou-se a interpolação pelo método de krigagem ordinária para estimar valore em locais não medidos. Com o uso do Idrisi 32 realizou-se uma reclassificação dos atributos químicos por quartis e para a produtividade usou a média, dividindo a área em duas classes: acima e abaixo da média e, posteriormente, calculado a porcentagem de cada classe.

\section{RESULTADOS E DISCUSSÃO}

Os resultados da análise descritiva para os atributos químicos e a produção de pimenta-doreino estão apresentados na Tabela 1 .

Comparando os valores médios dos atributos com as classes de interpretação da fertilidade para o Estado do Espírito Santo - Brasil (Ribeiro et al., 1999), verifica-se baixos valores para $\mathrm{pH}, \mathrm{Ca}, \mathrm{SB}$, $\mathrm{V}$ e médios para $\mathrm{K}, \mathrm{Mg}$ e CTC. Nesse caso, esses valores são esperados, pois a amostragem do solo ocorreu após a colheita da cultura.

Todos os atributos apresentaram assimetria positiva, com a média maior que a mediana dos dados, o que indica existência de uma elevada freqüência de valores abaixo da média, com exceção para o $\mathrm{H}+\mathrm{Al}$. A análise de normalidade dos dados pelo teste de Kolmogorov-Smirnov-KS revelou que os atributos $\mathrm{pH}, \mathrm{P}, \mathrm{Al}, \mathrm{V}, \mathrm{H}+\mathrm{Al}$ e produção seguiram a distribuição normal padrão. De acordo com Cressie (1991), a normalidade dos dados não é uma exigência da geoestatística, é conveniente apenas que no gráfico de distribuição normal o atributo não apresente caudas muito alongadas, o que poderia comprometer as análises. Mais importante que a normalidade dos dados é a ocorrência ou não do chamado efeito proporcional, em que a média 
Tabela 1

Análise descritiva dos atributos químicos do solo, na profundidade de 0-0,20 m, e produção da cultura pimenta-do-reino

\begin{tabular}{|l|l|c|c|c|c|c|c|c|c|c|}
\hline \multirow{2}{*}{ Atributos } & \multicolumn{4}{|c}{} & \multicolumn{2}{c|}{ Valores } & \multicolumn{3}{c|}{ Coeficientes } & Teste \\
\cline { 2 - 12 } & \multicolumn{1}{|c|}{ Unidade } & Med & Md & S & Mín. & Máx. & CV $(\%)$ & Ck & Cs & KS \\
\hline $\mathrm{pH}$ & $(\mathrm{em} \mathrm{água})$ & 4,7 & 4,7 & 0,6 & 3,7 & 6,6 & 10,9 & 0,26 & 0,50 & $\mathrm{~ns}$ \\
\hline $\mathrm{P}$ & $\left(\mathrm{mg} \mathrm{dm}^{-3}\right)$ & 32,1 & 29,0 & 18,5 & 8,0 & 76,0 & 54,0 & 0,46 & 0,83 & $\mathrm{~ns}$ \\
\hline $\mathrm{K}$ & $\left(\mathrm{mg} \mathrm{dm}^{-3}\right)$ & 69,0 & 56,0 & 55,8 & 22,0 & 198 & 58,5 & 1,92 & 1,57 & $*$ \\
\hline $\mathrm{Ca}$ & $\left(\mathrm{cmol}_{\mathrm{c}} \mathrm{dm}^{-3}\right)$ & 0,77 & 0,60 & 0,69 & 0,2 & 2,0 & 54,6 & 3,27 & 1,51 & $*$ \\
\hline $\mathrm{Mg}$ & $\left(\mathrm{cmol}_{\mathrm{c}} \mathrm{dm}^{-3}\right)$ & 0,52 & 0,50 & 0,29 & 0,2 & 1,1 & 47,5 & 0,39 & 0,90 & $*$ \\
\hline $\mathrm{Al}$ & $\left(\mathrm{cmol}_{\mathrm{c}} \mathrm{dm}^{-3}\right)$ & 0,51 & 0,50 & 0,31 & 0,1 & 1,4 & 57,3 & $-0,12$ & 0,35 & $\mathrm{~ns}$ \\
\hline $\mathrm{SB}$ & $\left(\mathrm{cmol}_{\mathrm{c}} \mathrm{dm}^{-3}\right)$ & 1,50 & 1,40 & 0,94 & 0,7 & 3,4 & 38,6 & 1,10 & 1,02 & $*$ \\
\hline $\mathrm{CTC}$ & $\left(\mathrm{cmol}_{\mathrm{c}} \mathrm{dm}^{-3}\right)$ & 5,64 & 5,60 & 1,24 & 4,0 & 7,9 & 13,6 & 0,32 & 0,17 & $*$ \\
\hline V & $(\%)$ & 27,4 & 25,4 & 14,5 & 11,6 & 52,6 & 37,4 & $-0,30$ & 0,07 & $\mathrm{~ns}$ \\
\hline $\mathrm{H}+\mathrm{Al}$ & $\left(\mathrm{cmol}_{\mathrm{c}} \mathrm{dm}^{-3}\right)$ & 3,91 & 4,05 & 0,98 & 1,5 & 5,9 & 25,6 & 2,96 & $-0,54$ & $\mathrm{~ns}$ \\
\hline Produção & $\mathrm{kg} \mathrm{planta}^{-1}$ & 2,70 & 2,45 & 1,40 & 0,5 & 6,4 & 52,0 & $-0,48$ & 0,50 & $\mathrm{~ns}$ \\
\hline
\end{tabular}

1 cachos verdes; Med:- média; Md - mediana; S - desvio padrão; Máx.-máximo; Mín.-mínimo; CV - coeficiente de variação; Cs - coeficiente de simetria; Ck - coeficiente de curtose; ns- distribuição normal pelo teste Kolmogorov-Smirnov-KS a 5\% de probabilidade; * - distribuição não definida.

e a variabilidade dos dados sejam constantes na área em estudo, ou seja, ocorrer a estacionariedade necessária ao uso da geoestatística (Isaaks \& Srivastava, 1989).

Segundo Landim (2003), o coeficiente de variação $(\mathrm{CV})$ fornece uma medida relativa da precisão do experimento, sendo bastante útil na avaliação da dispersão dos dados. Portanto, os CV foram classificados segundo os critérios estabelecidos por Warrick \& Nielsen (1980). Sendo assim, apresentou baixa variabilidade para o $\mathrm{pH}$ $(<12 \%)$ e moderada para os demais atributos, entre $12 \%$ a $60 \%$. A presença de elevados coeficientes de variação pode demonstrar grandes alterações provocadas pelo manejo do solo como na adubação, em calagens sucessivas e irregulares e, bem como, o local de amostragem. Os atributos que apresentaram os maiores CV têm uma grande amplitude entre o valor mínimo e o máximo, com uma relação em torno de 10 vezes.

Esse fato pode ser entendido examinando-se os valores mínimos $\left(0,2 \mathrm{mg} \mathrm{dm}^{-3}\right)$ e máximos $(2,2 \mathrm{mg}$ $\mathrm{dm}^{-3}$ ) do Ca. Nota-se que, além de apresentar um quadro nutricional bastante variável, a área do estudo está necessitando de calagem, com base nos valores encontrados. Os atributos $\mathrm{SB}, \mathrm{V}, \mathrm{CTC}$ e $\mathrm{Mg}$ apresentaram média variabilidade, concordando com os valores encontrados no plantio de soja por Manzione (2002).

O K apresentou o maior CV $(58,5 \%)$, bem próximo de $58,2 \%$ e $50 \%$ encontrados por Carvalho et al. (2002) e Oliveira (2007), respectivamente. A textura do solo na camada de 0-0,2 m é francoarenosa o que pode ter influenciado na variabilidade e na quantidade de K em alguns pontos. Segundo Braga (1994), a pobreza de K será maior em solos arenosos devido a sua grande mobilidade. Nesse caso, demonstra que este solo não tem capacidade de suprimento de potássio, assim como, o potássio trocável não é suficiente para sustentar cultivos por períodos prolongados na área, necessitando de reposição em espaço de tempo menor.

No Brasil, a necessidade de nutrientes por planta adulta de pimenta-do-reino, para sua manutenção e produção foi estimada em $90 \mathrm{~g}$ de $\mathrm{N}, 10 \mathrm{~g}$ de $\mathrm{P}$, $120 \mathrm{~g}$ de K, $80 \mathrm{~g}$ de Ca e $11 \mathrm{~g}$ de $\mathrm{Mg}$ (Kato, 1978). Os valores médios encontrados para o $\mathrm{Ca}, \mathrm{Mg}$ e $\mathrm{K}$ se encontram bem abaixo da necessidade por planta 
adulta para sua manutenção e produção. Para esses elementos verificou-se grande amplitude entre os dados, indicando que o maior valor do elemento encontrado no solo, apresenta-se bem abaixo do valor recomendado.

Conforme se espera, verificou-se correlação alta e positiva do valor de $\mathrm{pH}$ com a CTC total e a SB (Figura 1), pois com o aumento do valor do $\mathrm{pH}$ há liberação de sítios de troca negativo dos colóides do solo e disponibilidade de cátions básicos fornecidos com o ânion básico acompanhante. Analogamente, verificou-se correlação alta e negativa do valor de $\mathrm{pH}$ com a acidez potencial $(\mathrm{H}+\mathrm{Al})$ e com a quantidade de $\mathrm{Al}$ livre, pois com a redução da acidez ativa mais $\mathrm{Al}$ é precipitado e mais hidrogênio se torna indisponível, ficando nos sítios de troca, antes ocupados por $\mathrm{He}$ Al, os cátions básicos (Conde, 2000).

$\mathrm{Na}$ análise geoestatística, com a finalidade de comparar os semivariogramas, e conseqüentemente a variabilidade espacial de cada atributo do solo sob cultivo da pimenta-do-reino, utilizou-se da técnica de escalonamento da semivariância pela variância dos dados para facilitar a sua interpretação em uma escala padronizada, como utilizado por Vieira et al. (1991), estão apresentados na Tabela 2.
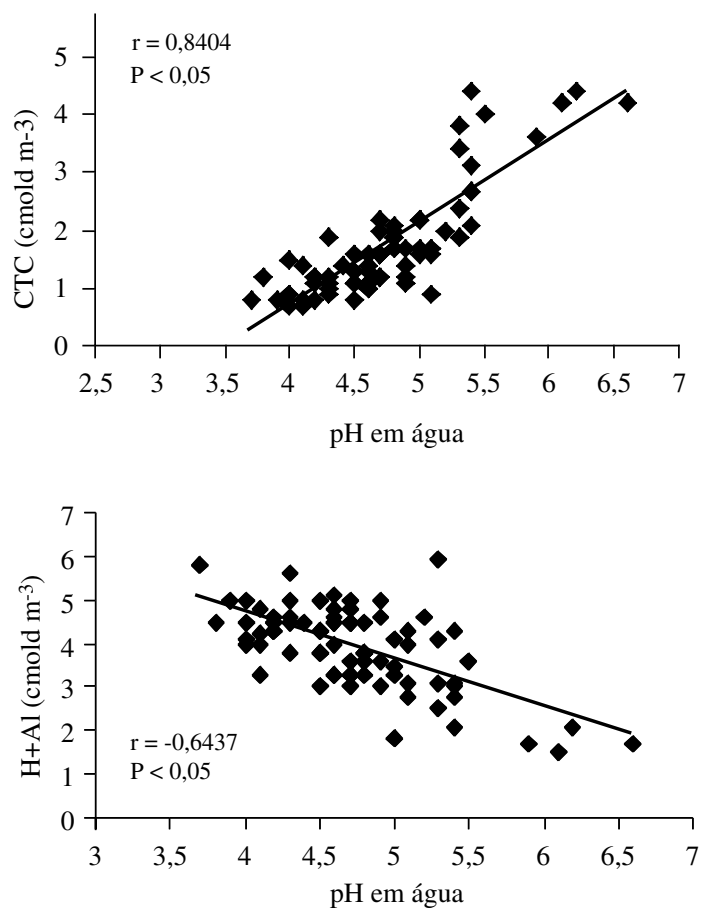

Para os atributos $\mathrm{Ca}, \mathrm{Mg}, \mathrm{SB}, \mathrm{V}$ e CTC não foram encontrados ajustes do semivariograma experimental a um modelo teórico, apresentando, assim, efeito pepita puro (EPP) ou ausência total de dependência espacial para distâncias maiores que a menor distância adotada entre as amostras. Segundo Vieira (2000) o EPP significa que o alcance, para os atributos em questão, é menor que o menor espaçamento entre as amostras. De acordo com Silva (1988), esses atributos têm uma distribuição completamente aleatória e a única estatística aplicável é a estatística clássica. Porém, foi verificado por Vieira (1997) em seu estudo, que esses mesmos atributos apresentaram dependência espacial e o modelo ajustado foi o esférico. Como se sabe, a ocorrência da dependência espacial depende de fatores, tais como: cultura instalada, manejo do solo e da planta, topografia, textura do solo, clima, insumos utilizados, escala de amostragem e outros.

Os demais atributos químicos mostraram dependência espacial, com alcance variando entre 3,8 a 15,7 m e com ajuste ao modelo esférico para o $\mathrm{pH}$ e $\mathrm{H}+\mathrm{Al}$, respectivamente. Os atributos $\mathrm{P}, \mathrm{Ke} \mathrm{Al}$ ajustaram ao modelo exponencial. Segundo Journel \& Huijbregts (1991), o alcance da dependência
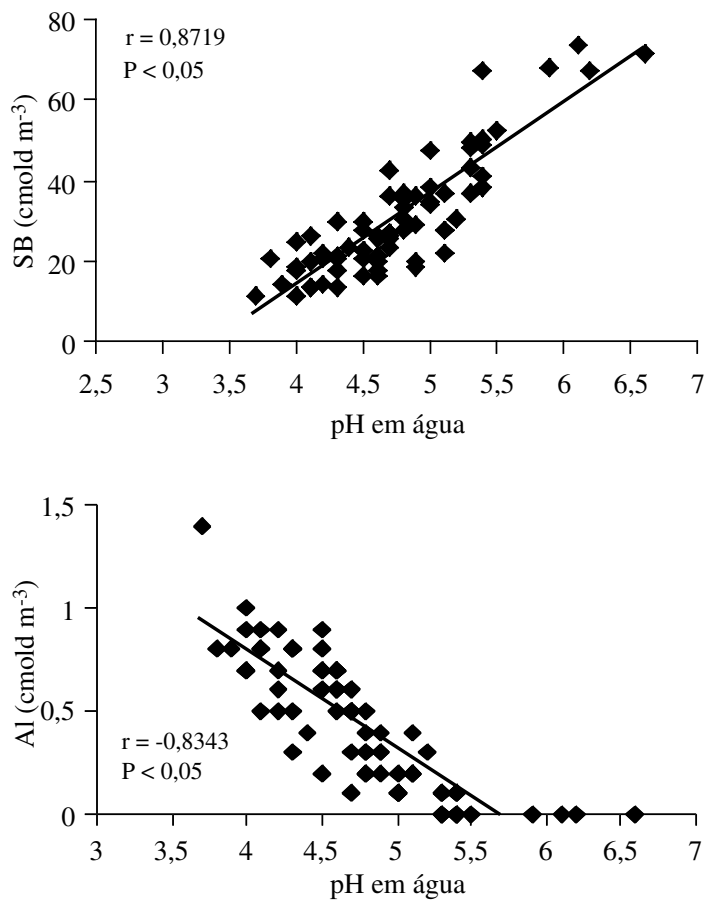

Figura 1. Correlação linear entre o valor de $\mathrm{pH}$ em água e a capacidade de troca de cátions (CTC), a soma de cátions básicos (SB), a acidez potencial $(\mathrm{H}+\mathrm{Al})$ e o alumínio do solo. 
Tabela 2

Parâmetros dos modelos dos semivariogramas ajustados aos dados de solo e produção de pimenta-do-reino

\begin{tabular}{|l|c|c|c|c|c|c|c|}
\hline \multicolumn{1}{|c|}{ Variável } & Modelo Ajustado & $\mathbf{a}(\mathbf{m})$ & $\mathbf{C}_{\mathbf{0}}+\mathbf{C}_{\mathbf{1}}$ & $\mathbf{C}_{\mathbf{0}}$ & IDE $(\boldsymbol{\%})$ & $\mathbf{R}^{\mathbf{2}}$ & Classe Espacial \\
\hline $\mathrm{pH}$ & $\mathrm{ESF}$ & 4,7 & 0,91 & 0,20 & 78 & 60 & Forte \\
\hline $\mathrm{P}$ & $\mathrm{EXP}$ & 15,7 & 1,07 & 0,51 & 52 & 92 & Moderada \\
\hline $\mathrm{K}$ & $\mathrm{EXP}$ & 7,0 & 1,04 & 0,31 & 70 & 90 & Moderada \\
\hline $\mathrm{Ca}$ & $\mathrm{EPP}$ & - & 0,88 & 0,88 & - & - & - \\
\hline $\mathrm{Mg}$ & $\mathrm{EPP}$ & - & 0,88 & 0,88 & - & - & - \\
\hline $\mathrm{Al}$ & $\mathrm{EXP}$ & 6,5 & 1,0 & 0,31 & 70 & 54 & Moderada \\
\hline $\mathrm{SB}$ & $\mathrm{EPP}$ & - & 0,77 & 0,77 & - & - & - \\
\hline $\mathrm{CTC}$ & $\mathrm{EPP}$ & - & 1,1 & 1,1 & - & - & - \\
\hline $\mathrm{V}$ & $\mathrm{EPP}$ & - & 0,93 & 0,93 & - & - & - \\
\hline $\mathrm{H}+\mathrm{Al}$ & $\mathrm{ESF}$ & 3,80 & 0,93 & 0,21 & 77 & 42 & Forte \\
\hline Produção & ESF & 4,20 & 0,95 & 0,23 & 76 & 51 & Forte \\
\hline
\end{tabular}

IDE - Índice de dependência espacial $C_{1} /\left(C_{0}+C_{1}\right) ; a-a l c a n c e ; C_{0}$-efeito pepita; $C_{0}+C_{1}$ - Patamar; ESF- modelo esférico; EXP- modelo exponencial e EPP- modelo efeito pepita puro.

espacial representa a distância em que os pontos amostrais estão correlacionados entre si, ou seja, os pontos localizados numa área de raio igual ao alcance são mais parecidos entre si do que com aqueles localizados fora desta área. Segundo Trangmar et al. (1985), o seu conhecimento é importante na definição de um número ótimo de sub-amostras coletadas, além de aumentar a representatividade da amostra. Neste sentido, Mulla \& McBratney (2000) recomendam que a distância entre pontos amostrais seja de 0,25 a 0,50 do valor do alcance do atributo sob análise.

Com base no IDE (índice de dependência espacial), os atributos $\mathrm{pH}, \mathrm{H}+\mathrm{Al}$ e produção apresentaram forte dependência espacial e com média dependência para $\mathrm{P}, \mathrm{K}$ e Al. Em relação aos parâmetros dos semivariogramas ajustados, notase que com exceção do $\mathrm{H}+\mathrm{Al}$, todos os modelos foram enquadrados nos critérios de aceitação, que foram $\mathrm{R}^{2}$ do semivariograma igual ou maior que 0,50 e $\mathrm{R}^{2}$ significativo a $5 \%$ na validação cruzada (Azevedo, 2004).

A produção de pimenta-do-reino, $\mathrm{pH}$ e $\mathrm{H}+\mathrm{Al}$ apresentaram semivariogramas muito parecidos, com valores de alcance muito próximos 4,7; 3,8 e $4,2 \mathrm{~m}$, respectivamente. Esse fato pode indicar que a produção na área esta associada com a disponibilidade de $\mathrm{H}$ e $\mathrm{Al}$ na solução (toxidez de alumínio). No caso do calcário que, geralmente, é distribuído uniformemente sobre o solo, não apresentou variabilidade espacial para o $\mathrm{Ca}$ e o $\mathrm{Mg}$. Porém, o P e o K são distribuídos no solo na projeção da copa da cultura, proporcionando maior continuidade espacial para o P com um alcance de $15,7 \mathrm{~m}$. A variabilidade espacial de $\mathrm{P}$ pode ser explicada pela sua baixa mobilidade no solo, enquanto que, o K a sua alta mobilidade esta relacionada com a textura do solo na área, como discutido anteriormente, e pela localização das plantas.

Concordando com Azevedo (2004), a geoestatística permitiu detectar a existência de uma estrutura de dependência espacial e com os parâmetros do semivariograma estimar por krigagem ordinária valores em locais não medidos. Foi possível mapear e identificar os locais e suas respectivas representações de valores, como pode ser visto nas Figuras 2, 3 e 4.

Em relação à produção, verificou-se que 49,93\% da área apresentou valor menor que a média de $2,7 \mathrm{~kg}$ planta $^{-1}$, indicando um baixo valor em comparação com outros produtores da região (Figura 2).

Nesse propósito, o mapa da distribuição espacial de atributos químicos na área permitiu localizar problemas e a possibilidade de sugerir práticas ao 


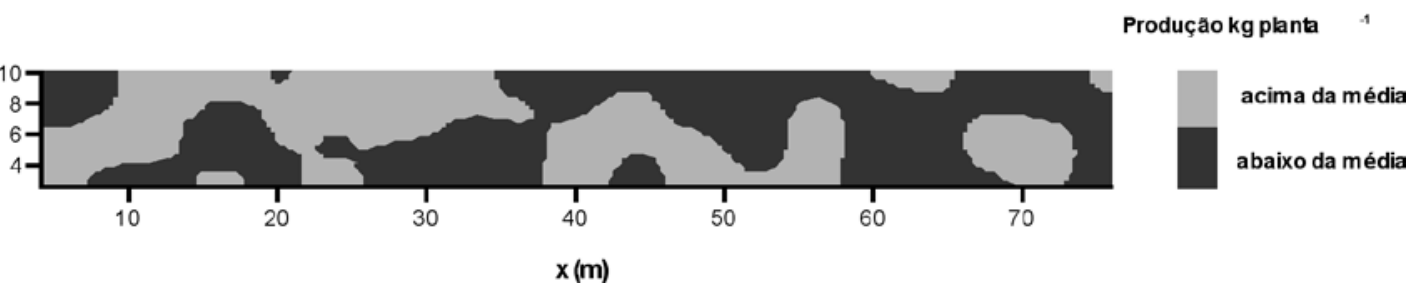

Figura 2. Mapa de produção de pimenta-do-reino, normalizada pela média, dividido em duas classes: acima e abaixo da média.

$\mathbf{a}$

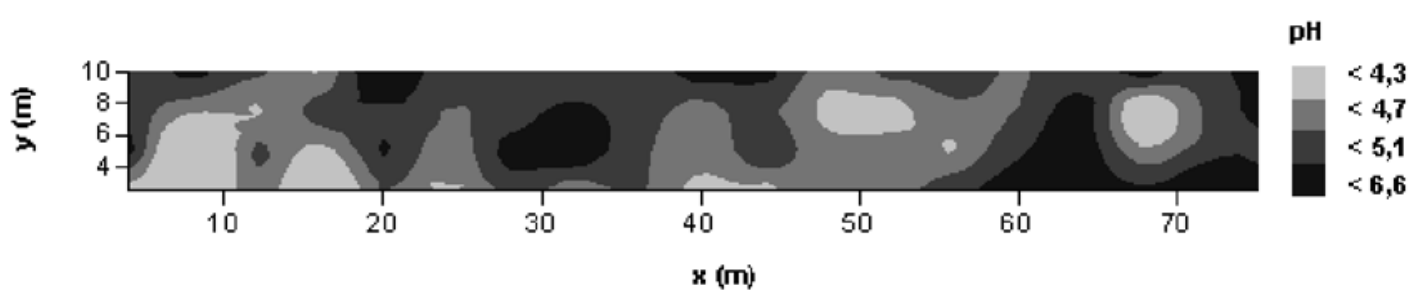

b

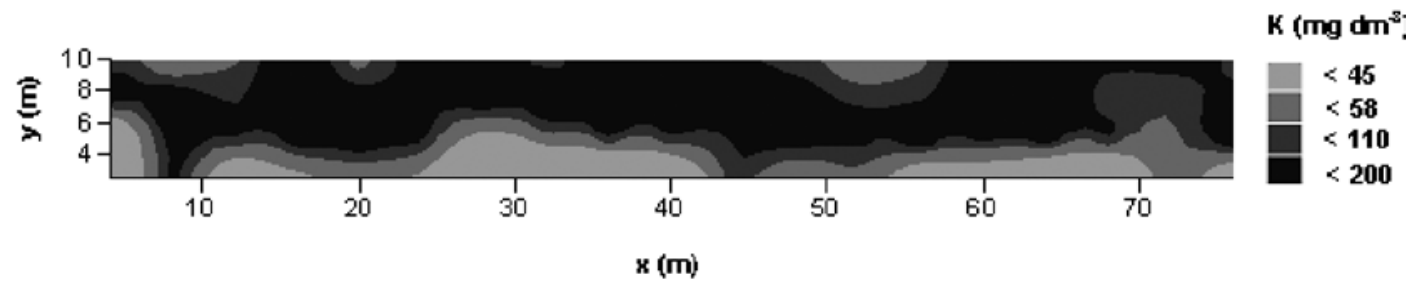

c

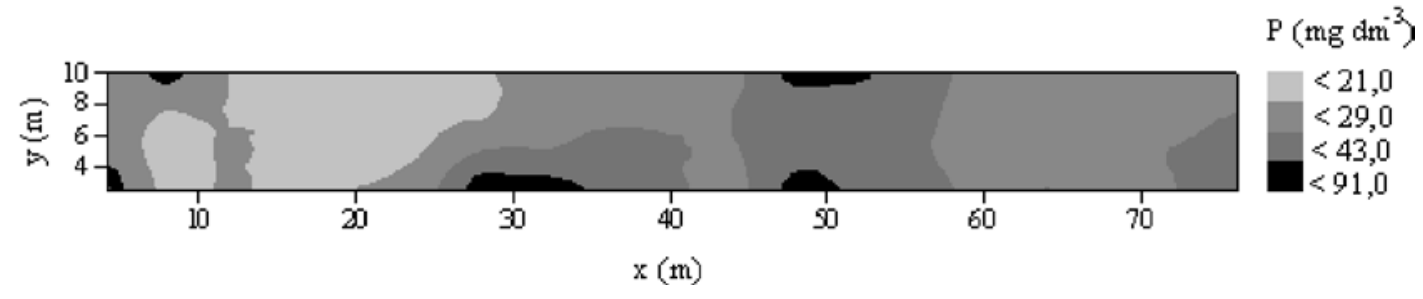

Figura 3. Mapa classificado por quartis para: a- acidez ativa (pH), b- potássio (K) e c- fósforo.

agricultor segundo as necessidades apresentadas, bem como a aplicação de insumos a taxas variáveis, na tentativa de solucioná-los.

Observa-se uma grande amplitude nos atributos químicos estudados. Esta grande amplitude revela os problemas que podem ocorrer quando se usa a média dos valores para o manejo da fertilidade. Em alguns locais da área, a aplicação de fertilizante será inferior à dosagem necessária; em outros, a aplicação será de acordo com as necessidades e, em outros, poderá haver aplicação excessiva.

Considerando que a cultura de pimenta-doreino procura atingir, pela calagem do solo, uma saturação de bases (V) de $70 \%$, observa-se que o valor médio encontrado $(27,4 \%)$ está bem abaixo do desejado. Com a sua distribuição espacial (Figura 4a), observa-se nesse solo grande necessidade de calagem ao longo da área e de forma diferenciada. Caso a 
a

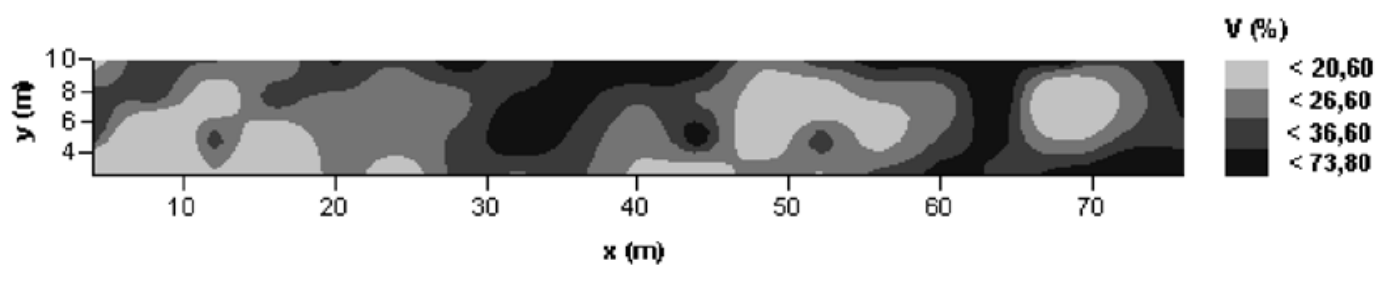

b

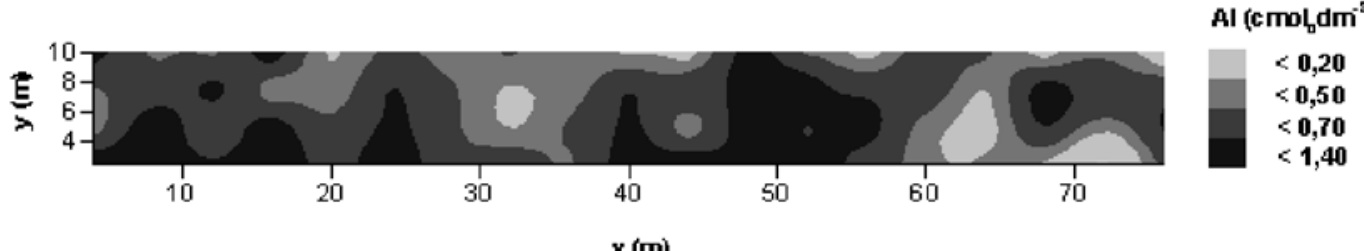

c

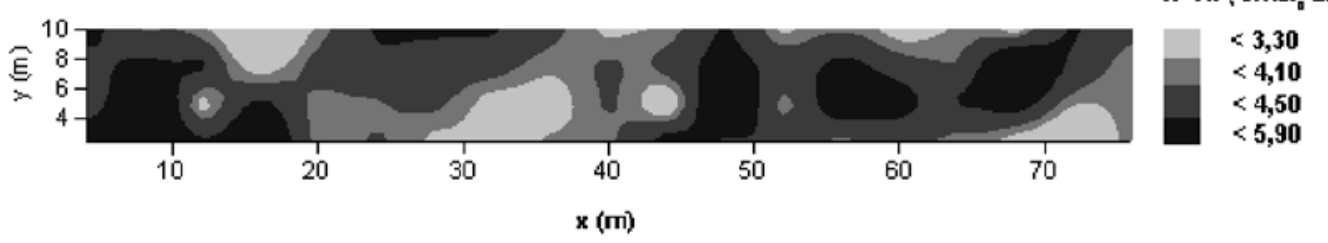

Figura 4. Mapa classificado por quartis para: a- saturação por bases (V\%), b- acidez trocável (Al) e c- acidez potencial (H+Al).

aplicação do corretivo não for efetuada considerando a variabilidade da área, a produção da cultura não chegará ao potencial desejado e possivelmente em toda a área, principalmente, devido à acidez ativa e a potencial. Estes atributos interferem diretamente na disponibilidade de $\mathrm{Al}$ e de cátions básicos.

\section{CONCLUSÕES}

Com a análise dos resultados concluiu-se que as técnicas de geoestatística mostraram-se aplicáveis no diagnóstico de atributos químicos do solo e da

\section{LITERATURA CITADA}

AZEVEDO, E.C. 2004. Uso da geoestatística e de recursos de geoprocessamento no diagnóstico da degradação de um solo argiloso sob pastagem no Estado de Mato Grosso. Tese (Doutorado), Faculdade de Engenharia Agrícola de Campinas, $158 \mathrm{f}$.

BRAGA, J.M. 1994. Potássio. Modulo VIII. Curso de Fertilidade e Manejo do solo. (Nacional). Referências adicionais: Brasil/Português: Meio de divulgação: Impresso. produção da pimenta-do-reino, o que permitiu o seu mapeamento. Os atributos $\mathrm{pH}, \mathrm{K}, \mathrm{Al}$ e $\mathrm{H}+\mathrm{Al}$ apresentaram valores baixos de alcance, com exceção do $\mathrm{P}$, evidenciando a baixa continuidade do solo sob cultivo da pimenta-do-reino. A grande amplitude apresentada pelos valores encontrados justifica a aplicação diferenciada e localizada de fertilizantes na lavoura e que o método convencional de amostragem de solo para análise química, baseado no valor médio, pode ser aplicado para a correção da acidez na área, de acordo com a não caracterização da dependência espacial apresentada para o $\mathrm{Ca}$ e $\mathrm{Mg}$.

CARVALHO, J. R.P.; SILVEIRA, P.M.; VIEIRA, S.R. 2002. Geoestatística na determinação da variabilidade espacial de características químicas do solo sob diferentes preparos. Pesq. agropec. bras., Brasília, v. 37, n. 8, p. 1151-1159.

CONDE, R.P. 2000. Geoestatística aplicada a avaliação de reservas e controle de lavra na mina de cana brava (GO). Tese (Doutorado em Recursos Minerais e Hidrogeologia), Instituto de Geociências-Universidade de São Paulo. São Paulo, $161 \mathrm{p}$.

CRESSIE, N. 1991. Statistics for spatial data. New York: John Wiley. 
EMBRAPA. 1997. Centro Nacional de Pesquisa de Solos. Manual de métodos de análise de solo. 2.ed. Rio de Janeiro, $212 \mathrm{p}$.

EMBRAPA. 1999. Centro Nacional de Pesquisa de Solos. Sistema Brasileiro de Classificação de Solos. Rio de Janeiro. $412 \mathrm{p}$.

GANDAH, M. ET AL. 2000. Dynamics of spatial variability of millet growth and yields at three sites in Niger, west Africa and implications for precision agriculture research. Agricultural Systems, Oxon, v. 63, n. 2, p. 123-140

GONÇALVES, A.C.A.; FOLEGATTI, M.V.; MATA, J.D.V. 2001. Análises exploratória e geoestatística da variabilidade de propriedades físicas de um Argissolo Vermelho. Maringá Acta Scientiarum. V.23, n. 5, p. 1149-1157.

HOAGLIN, D.C.; MOSTELLER, F.; TYKEY, J.W. 1983 Análise exploratória de dados: técnicas robustas, um guia. Lisboa: Editora Salamandra, 446 p.

ISAAKS, E.H.; SRIVASTAVA, R.M. 1989. Applied geoestatistics: introduction to applied geostatistics. Oxford: University Press, $561 \mathrm{p}$.

JOURNEL, A.G.; HUIJBREGTS, C.J. 1991. Mining geostatistics. London, Academic Press, 600 p.

KATO, A.K. 1978. Teor e distribuição de $\mathrm{N}, \mathrm{P}, \mathrm{K}, \mathrm{Ca}$ e $\mathrm{Mg}$ em pimenteiras do reino (Piper nigrum, L.), 1978. 75f (Dissertação de Mestrado). Escola Superior de Agricultura "Luiz de Queiroz", Universidade de São Paulo.

LANDIM, P.M.B. 2003. Análise estatística de dados geológicos. 2. ed. São Paulo. UNESP, 253 p.

LIMA, J.S.S.; OLIVEIRA, R.B.; QUARTEZANI, W.Z. 2007. Variabilidade espacial de atributos físicos de um latossolo vermelho-amarelo sob cultivo de pimenta-doreino. Engenharia na Agricultura, Viçosa, v. 15, n. 3, p. 290-298.

MANZIONE, R.L. 2002. Variabilidade espacial de atributos químicos do solo em Araguari-MG. 2002. 141f. (Dissertação de Mestrado). UNESP-SP.

MULLA, D.J.; MCBRATNEY,A.B. 2000. Soil spatial variability. In: SUMNER, M. E. Handbook of soil science. Boca Raton: CRC Press, P. A 321-352.

OLIVEIRA, R.B. 2007. Mapeamento e correlação de atributos do solo e de plantas café conilon para fins de agricultura de precisão. 2007. 129f. (Dissertação de Mestrado). Universidade Federal do Espírito Santo.

ORTIZ, J.L. 2003. Emprego do geoprocessamento no estudo da relação entre potencial produtivo de um povoamento de eucalipto e atributos do solo e do relevo. (Dissertação de Mestrado). Escola Superior de Agricultura "Luiz de Queiroz”, Universidade de São Paulo, $205 f$.

ROBERTSON, G.P. 1998. GS+: Geoestatistics for the environmental sciences-GS ${ }^{+}$User's Guide. Plainwell, Gamma Desing Software, $152 \mathrm{p}$.

RIBEIRO, C.A.; GUIMARÃES, P.T.G.; ALVAREZ, V.V.H. 1999. Recomendações para o uso de corretivos e fertilizantes em Minas Gerais. $5^{\text {a }}$ aproximação. Viçosa-MG, 359 p.

SARAIVA A.M.; CUGNASCA, C.E.; HIRAKAWA, A.R. 2000. Aplicação em taxa variável de fertilizantes e sementes. In: BORÉM, A.; GIÚDICE, M.P.; QUEIROZ, D.M. MANTOVANI, E.C.; FERREIRA, L.R.: VALLE, F.X.R.; GOMIDE, R.L. Agricultura de precisão. Viçosa: UFV.

SECUNDINO, W. (COORD.). 2003. Pimenta-do-reino. São Mateus. INCAPER, 2003. Disponível em: <www.incaper. es.gov.br/pedeag/pimenta-seminario.htm.>. Acesso em: 28 de maio 2004.

SILVA, A.P. 1988. Variabilidade especial de atributos físicos do solo. Tese (Doutorado)-ESALQ-USP, Piracicaba, 105 p.

VELOSO, C.A.C.; MURAOKA, T. 1993. Diagnosis of macronutrient deficiency symptoms in black pepper (Piper nigrum, L.). Sci. Agric. (Piracicaba, Braz.), v. 50, n. 2, p. $232-236$.

TRANGMAR, B.B.; YOST, R.S.; UEHARA, G. 1985. Application of geostatistics to spatial studies of soil properties. Advances in Agronomy, V. 38, p. 45-94.

VIEIRA, S.R. 1997. Variabilidade especial de argila, silte e atributos químicos em uma parcela experimental de um Latossolo Roxo de Campinas (SP). Bragantia, v. 56, n. 1, p. 181-190.

VIEIRA, S.R. 2000. Geoestatística em estudos de variabilidade espacial do solo. In. NOVAES, R. F.; ALVAREZ V, V. H.; SCHAEFER, C. E G. R. Tópicos em ciências do solo, Viçosa: Sociedade Brasileira de Ciência do Solo. v. 1, p. 2-54.

VIEIRA, S.R.; HATFIELD, J.L.; NIELSEN, D.R.; BIGGAR, J.W.1983. Geostatistical theory and application to variability of some agronomical properties. Hilgardia, Oakland, v. 51.

WARRICK, A.W.; NIELSEN, D.R. . 1980. Spatial variability of soil physical properties in the field. In: HILLEL, D., ed. Application of soil physics. New York: Academic Press. p. 319-324.

ZIMBACK, C.R.L. 2001. Análise espacial de atributos químicos de solos para fins de mapeamento da fertilidade do solo. Tese (Livre-Docência)-Faculdade de Ciências Agronômicas, Universidade Estadual Paulista, Botucatu, SP. $114 \mathrm{f}$ 
